\title{
QUALIDADE DE VIDA, AUTO ESTIMA E AUTO IMAGEM DE JAPONESES DO BRASIL E DO JAPÃO
}

\section{Adriana Coutinho de Azevedo Guimarães}

Universidade do Estado de Santa Catarina, Florianópolis, Santa Catarina, Brasil

\section{André Manabu Kaneoya}

Universidade do Estado de Santa Catarina, Florianópolis, Santa Catarina, Brasil

\section{Zenite Machado}

Universidade do Estado de Santa Catarina, Florianópolis, Santa Catarina, Brasil

\section{Sabrina Fernandes}

Universidade Federal de Santa Catarina, Florianópolis, Santa Catarina, Brasil

\section{Amanda Soares}

Faculdade Porto das Águas, São José, Santa Catarina, Brasil

Resumo: Objetivo: Analisar e comparar a qualidade de vida (QV), auto estima e auto imagem de descendentes japoneses de Santa Catarina (SC) e de Yamanashi. Métodos: Uma amostra de 118

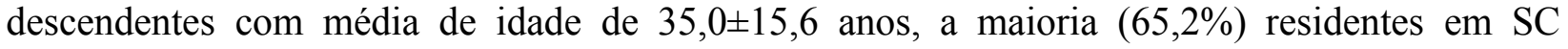
(Brasil). Resultados: Os sujeitos vivem sozinhos, tem trabalho remunerado e curso superior incompleto. A QV foi considerada de boa a muito boa (56-72\%), os descendentes de SC obtiveram escores superiores, com diferença significativa no domínio psicológico $(p=0,050)$. A auto estima, auto imagem obtiveram conceito baixo. Além disso, não possuem o hábito de praticar atividade física (AF) regular. Conclusão: A auto estima e auto imagem foram consideradas baixas, a QV boa, não diferindo entre os grupos de uma forma geral.

Palavras-chave: Japoneses, Qualidade de Vida, Imagem Corporal, Auto imagem

\section{Introdução}

A questão de maior tradição e que nunca recebeu uma resposta completa é o "por que as pessoas migram?" Há diversos fatores que desencadeiam essa migração, e a busca de melhor condição de vida é uma delas (MARTINELLO e KLUG, 2007). Especificamente sobre a imigração dos japoneses para o Brasil o fato desencadeador foi à expulsão dos japoneses do seu país por conflitos armados, a busca por proteção e segurança, além da atração exercida pelas condições socioeconômicas brasileiras. O marco dessa imigração foi à chegada da embarcação Kasato-Maru em 1908. Deu-se então o processo de formação de colônias em terras brasileiras e o objetivo era manter as tradições, a cultura e a educação para seus descendentes assim como no

Pensar a Prática, Goiânia, v. 14, n. 1, p. 1-12, jan./abr. 2011 
país de origem (TAKEUCHI, 2008). Entre os anos de 1953 e 1973 o contingente Japonês no Brasil era cerca de 53 mil pessoas que se concentraram mais nos estados de São Paulo, Paraná, Mato Grosso do Sul, Pará e Amazonas. O estado de São Paulo recebeu o maior contingente, cerca de $80 \%$ do total de imigrantes (PINHEIRO e SUAREZ, 2009).

Após a fixação dos imigrantes japoneses em território brasileiro misturando suas tradições à influência cultural nacional, contudo, mantiveram firme suas tradições, formando suas colônias e repassando aos seus descendentes suas origens (TAKEUCHI, 2008). Passado aproximadamente meio século os descendentes japoneses resolveram fazer o caminho inverso e buscaram no Japão uma oportunidade de melhorar suas condições e qualidade de vida. Em 2008 essa busca pela opção de vida no Japão cresceu vertiginosamente, não apenas por pequenos períodos de tempo, mas também com objetivo de fixar residência na terra do sol nascente.

Os brasileiros descendentes buscaram no Japão uma oportunidade de melhorar suas condições e qualidade de vida (TAKEUCHI, 2008). Mas o que seria uma busca por qualidade de vida? Há certa concordância ao definir que qualidade de vida é a percepção do indivíduo da sua posição na vida, no contexto da cultura e sistema de valores nos quais ele vive, e em relação aos seus objetivos, expectativas, padrões e preocupações. Trata-se de um conceito frequentemente utilizado para designar bem estar físico, psicológico, social e ambiental (THE WHOQOL GROUP,1995; FLECK e COL., 2008). O mesmo esta relacionado à auto estima, ao bem estar pessoal, abrangendo uma série de aspectos como a capacidade funcional, o nível socioeconômico, o estado emocional, a interação social, a atividade intelectual, o autocuidado, o suporte familiar, o estado de saúde geral, os valores culturais, éticos e a religiosidade (SANTOS e COL., 2002) e ainda, o estilo de vida, a satisfação com o emprego, às atividades diárias e o meio ambiente (VELARDE e ÁVILA, 2002; BOWLING e COL., 2003).

Como um dos componentes da qualidade de vida, a auto estima é definida como a imagem ou percepção que cada pessoa tem sobre seus valores e competências como ser humano. A mesma se expressa através de uma auto imagem positiva, estar satisfeito ou se aceitar como é, com suas limitações e habilidades pessoais, em termos de sua aparência física, comportamento e emoções. Ademais é uma importante variável para alcançar um nível adequado e uma melhor gestão social da vida (PEREIRA e COL., 2005). A atividade física colabora para a adoção de um estilo de vida saudável, refletindo sobre a qualidade de vida além de influenciar a auto estima e auto imagem (MAZO e COL., 2006).

Alguns estudos abordam a qualidade de vida (SOUSA e COL., 2003; ALEXANDRE e COL., 2009; NAGEL e COL., 2009), auto estima e auto imagem (KAKESHITA e ALMEIDA, 2006; MAZO e COL., 2006; COQUEIRO e COL., 2008). Porém, nesse processo de ida e vinda acredita-se que alguns fatores da qualidade de vida podem ser comprometidos. Buscou-se amparo na literatura para responder essa questão e observou-se a escassez de estudos sobre essa temática de forma que, este estudo objetivou analisar e comparar a qualidade de vida, auto estima e auto imagem de descendentes japoneses residentes nas colônias de Santa Catarina, nos municípios de São Joaquim, Frei Rogério, Caçador, Lages, Campo Belo do Sul, Joinville, Itajaí e Curitibanos (MARTINELLO e KLUG, 2007) e na província de Yamanashi, descendentes das cidades de Kofu, Fujiyoshida, Tsuru, Yamanashi, Otsuki, Nirasaki, Minamiarupusu, Hokuto, Kai, Fuefuki, Uenohara, Koshu e Chuo (PINHEIRO e SUAREZ, 2009).

Pensar a Prática, Goiânia, v. 14, n. 1, p. 1-12, jan./abr. 2011 


\section{Métodos}

O interesse desse estudo é comparar os imigrantes que se instalaram mais ao Sul do Brasil, especificamente no estado de Santa Catarina (São Joaquim, Frei Rogério, Caçador, Lages, Campo Belo do Sul, Joinville, Itajaí e Curitibanos) com os da região de Yamanashi descendentes das cidades de Kofu, Fujiyoshida, Tsuru, Yamanashi, Otsuki, Nirasaki, Minamiarupusu, Hokuto, Kai, Fuefuki, Uenohara, Koshu e Chuo no Japão.

De acordo com o último censo realizado pelo IBGE (2001) com a população de descendentes japoneses nomeadamente em Santa Catarina era de 332 indivíduos, já na província de Yamanashi residiam cerca de 4.348 brasileiros descendentes de japoneses. Os brasileiros formam a terceira maior comunidade estrangeira no Japão.

O estudo caracteriza-se por ser de corte transversal e foi formado por uma amostra total de 118 indivíduos. A amostragem foi não probabilística por conveniência de Santa Catarina (Brasil) e de Yamanashi (Japão) com idade superior a 18 anos e calculada com erro de $95 \%$ (BARBETTA, 2006).

Os dados foram coletados por meio de um questionário auto aplicável por correio e via internet pela distância das cidades (Brasil e Japão). Em algumas colônias, os questionários foram aplicados de forma presencial (Brasil). Os indivíduos foram informados sobre os objetivos do estudo e importância de suas respostas serem verdadeiras por meio do termo de consentimento livre e esclarecido que foi assinado pelos participantes. $\mathrm{O}$ instrumento foi respondido individualmente, garantindo seu devido sigilo e identidade dos participantes. Todas as questões foram bem explicadas através de um ofício (para aqueles que responderão via internet e correio) e todas as dúvidas solucionadas antes do início de seu preenchimento (quando na presença dos pesquisadores).

O instrumento foi dividido em três partes: a) Informações sociodemográficas; b) auto estima e auto imagem; c) qualidade de vida.

a) Informação sociodemográfica, foi constituído por questões sobre sexo, idade, estado conjugal, naturalidade, local onde reside, escolaridade, atividade profissional e estrato econômico (ABEP, 2008) para caracterizar a situação socioeconômica, classificando os descendentes japoneses em estratos econômicos: A, B, C, D e E;

b) Auto estima e auto imagem de Steglich (1978), composto por 78 questões específicas para a avaliação destes aspectos resultando em auto estima e auto imagem alta ou baixa e ainda dividindo-se em (orgânica, social, intelectual e emocional);

c) Qualidade de Vida - WHOQOL - abreviado (World Health Organization Quality of Life - WHOQOL) (FLECK e COL., 1999) dividido em quatro domínios: físico, psicológico, social e ambiental, onde os escores determinam a qualidade de vida nos domínios em 0 a 100, sendo que quanto mais próximo de 100, melhor é considerada a qualidade de vida. A versão em português do WHOQOL abreviado foi desenvolvida no Centro WHOQOL para o Brasil, no Departamento de Psiquiatria e Medicina Legal da Universidade Federal do Rio Grande do Sul em Porto Alegre no Estado do Rio Grande do Sul - Brasil sob a coordenação geral do Dr. Marcelo Pio de Almeida Fleck.

Uma das maiores dificuldades encontradas durante o estudo foi do alto número de recusas em participar, pois, após a leitura rápida houve o relato de excesso de informações pessoais e

Pensar a Prática, Goiânia, v. 14, n. 1, p. 1-12, jan./abr. 2011 
exposição destas que não era agradável compartilhar, comportamento característico desta população.

O estudo foi aprovado em 2009, sob protocolo 12/2009, pelo Comitê de Ética em Pesquisa da Universidade do Estado de Santa Catarina - UDESC, que segue as normas e diretrizes regulamentadoras da pesquisa envolvendo Seres Humanos - Resolução CNS 196/96.

Para a análise dos dados utilizou-se o software SPSS - versão 16.0. A fim de verificar a existência de diferença significativa entre os valores baixo e alto das variáveis relacionadas a auto estima e auto imagem optou-se pelo Teste do Qui - quadrado. Este mesmo teste foi utilizado para comparar sexo, escolaridade, estrato econômico com a qualidade de vida. Utilizou-se o Teste T para amostras independentes para verificar a diferença entre os escores da qualidade de vida entre os descendentes japoneses residentes nas colônias de Santa Catarina e dos residentes na província de Yamanashi (Japão). O nível de significância estabelecido para os testes foi de 95\%.

\section{Resultados}

Participaram do estudo 118 descendentes japoneses, sendo $77(65,2 \%)$ descendentes das

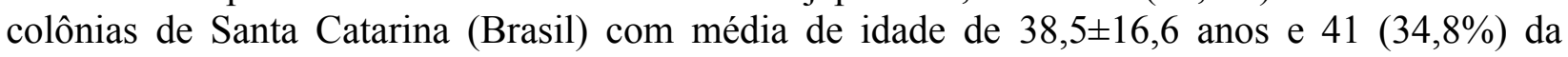

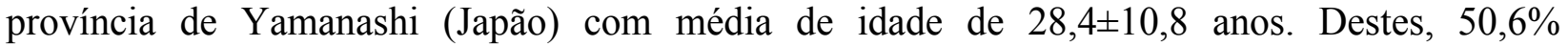
descendentes das colônias de Santa Catarina (Brasil) são do sexo feminino e 56,1\% da província de Yamanashi (Japão) são do sexo masculino, vivendo em sua maioria sozinhos, tendo trabalho remunerado, curso superior incompleto. No Brasil sobressai o estrato econômico A e B e no Japão C e D (tabela 1).

Tabela 1: Características pessoais dos descendentes japoneses

\begin{tabular}{llrrrr}
\hline Variáveis & $\mathrm{n}$ & \multicolumn{1}{c}{$\mathrm{SC}$} & $\mathrm{SC}(\%)$ & \multicolumn{1}{c}{ YM } & YM (\%) \\
\hline Sexo & 118 & 77 & & 41 & \\
Masculino & & 38 & 49,4 & 23 & 56,1 \\
Feminino & & 39 & 50,6 & 18 & 43,9 \\
Estado Civil & 117 & 77 & & 40 & \\
Vive com companheiro & & 36 & 46,8 & 7 & 17,5 \\
Vive sozinho & & 41 & 53,2 & 33 & 82,5 \\
Ativ. Ocupacional & 118 & 77 & & 41 & \\
Trabalho Remunerado & & 48 & 62,3 & 28 & 68,3 \\
Estudante & & 29 & 37,7 & 13 & 31,7 \\
Escolaridade & 118 & 77 & & 41 & \\
Superior Incompleto & & 41 & 53,2 & 33 & 80,5 \\
Superior Completo & & 36 & 46,8 & 8 & 19,5 \\
Estrato Economico & 118 & 77 & & 41 & \\
A e B & & 64 & 83,1 & 16 & 39,0 \\
C e D & & 13 & 16,9 & 25 & 61,0 \\
\hline SC
\end{tabular}

SC para Santa Catarina (Brasil) e YM para Yamanashi (Japão).

Pensar a Prática, Goiânia, v. 14, n. 1, p. 1-12, jan./abr. 2011 
Os descendentes japoneses investigados no presente estudo, independentemente do local de residência atual, apresentaram auto estima e auto imagem classificadas como baixa, como mostra a Tabela 2. De maneira similar, ambos os grupos manifestaram escores baixos em todas as subcategorias da auto imagem. Estes resultados foram comprovados através da observação de diferenças significativas nos testes aplicados. Da comparação inter grupos resultou que embora ambos os grupos apresentem escores baixos, os dos descendentes que residem no Japão são significativamente inferiores em todos os indicadores.

Tabela 2: Caracterização dos descendentes japoneses quanto à auto estima, auto imagem e suas categorias

\begin{tabular}{|c|c|c|c|c|c|}
\hline Variáveis & $\mathbf{N}$ & Total (\%) & $\mathrm{SC}(\%)$ & YM (\%) & Valor de p \\
\hline Auto estima & 118 & & & & 0,005 \\
\hline$\overline{\text { Alta }}$ & & 19,5 & 26,0 & 7,3 & \\
\hline Baixa & & 80,5 & 74,0 & 92,7 & \\
\hline Auto imagem & 118 & & & & 0,001 \\
\hline Alta & & 22,0 & 29,9 & 7,3 & \\
\hline Baixa & & 78,0 & 70,1 & 92,7 & \\
\hline Orgânica & 118 & & & & $<0,001$ \\
\hline Alta & & 30,5 & 40,3 & 12,2 & \\
\hline Baixa & & 69,5 & 59,7 & 87,8 & \\
\hline Social & 118 & & & & 0,006 \\
\hline$\overline{\text { Alta }}$ & & 32,2 & 40,3 & 17,1 & \\
\hline Baixa & & 67,8 & 59,7 & 82,9 & \\
\hline Intelectual & 118 & & & & 0,003 \\
\hline$\overline{\text { Alta }}$ & & 27,1 & 35,1 & 12,2 & \\
\hline Baixa & & 72,9 & 64,9 & 87,8 & \\
\hline Emocional & 118 & & & & 0,004 \\
\hline$\overline{\text { Alta }}$ & & 33,1 & 41,6 & 17,1 & \\
\hline Baixa & & 66,9 & 58,4 & 82,9 & \\
\hline
\end{tabular}

SC para Santa Catarina (Brasil) e YM para Yamanashi (Japão).

Com relação à frequência da prática de atividade física, percebeu-se que em Santa Catarina os descendentes se preocupam mais com a prática do que na província de Yamanashi. No entanto, constatou-se que em termos gerais, os descendentes não têm o hábito regular de praticar atividade física, sendo que a média foi de $1,3 \pm 1,5$ dias na semana e $29,7 \pm 38,7$ minutos por dia.

Sobre a influência da cultura japonesa no estilo de vida dos descendentes, $86,4 \%$ relataram que ela exerceu uma influência positiva, 3,4\% uma influência negativa e 10,2\% relataram que a cultura foi indiferente em sua forma de viver. Dentre os fatores abordados como positivos os principais foram a disciplina, a alimentação, o autocontrole, a paciência e os valores. Não houve diferença significativa entre os descendentes residentes em Santa Catarina ou em Yamanashi.

Pensar a Prática, Goiânia, v. 14, n. 1, p. 1-12, jan./abr. 2011 
A qualidade de vida dos descendentes em ambas as localidades (Tabela 3) foi considerada boa, de acordo com a escala que varia de 0 - 100, onde os escores médios ficaram entre 56 e $72 \%$ para os domínios em Santa Catarina e em Yamanashi. Em ambos os grupos o domínio social foi o que apresentou escores superiores (72,7 para Santa Catarina e 65,8 para Yamanashi). Os descendentes japoneses residentes em Santa Catarina obtiveram escores superiores em todos os domínios da qualidade de vida, embora tenha sido verificada diferença estatística apenas no domínio psicológico $(\mathrm{p}=0,050)$.

Tabela 3: Médias e desvio-padrão dos escores dos domínios da qualidade de vida dos descendentes japoneses de Santa Catarina (Brasil) e da província de Yamanashi (Japão)

\begin{tabular}{lrrr}
\hline \multicolumn{1}{c}{ Domínios } & SC & YM & p \\
\hline Físico & $59,3 \pm 11,3$ & $56,8 \pm 12,9$ & 0,398 \\
Psicológico & $66,9 \pm 8,5$ & $61,7 \pm 11,0$ & $0,050^{*}$ \\
Social & $72,7 \pm 17,2$ & $65,8 \pm 16,0$ & 0,559 \\
Ambiental & $68,3 \pm 15,4$ & $62,1 \pm 17,4$ & 0,090 \\
\hline
\end{tabular}

Teste T. SC para Santa Catarina e YM para Yamanashi (Japão).

Na tabela 4 são apresentados os resultados do teste do Qui-quadrado entre os domínios da qualidade de vida considerando-se as variáveis sexo, grau de escolaridade e estrato econômico. Não foram observadas diferenças significativas entre as mesmas, sendo que o valor de $\mathrm{p}$ variou de $\mathrm{p}=0,141-0,882$.

Tabela 4: Diferenças entre sexo, escolaridade e classe econômica dos descendentes japoneses de Santa Catarina (Brasil) e da província de Yamanashi (Japão) com os domínios da qualidade de vida

\begin{tabular}{lcccccccc}
\hline \multicolumn{1}{c}{ Variáveis } & \multicolumn{2}{c}{ Físico } & \multicolumn{2}{c}{ Psicológico } & \multicolumn{2}{c}{ Social } & \multicolumn{2}{c}{ Ambiental } \\
\hline & SC & YM & SC & YM & SC & YM & SC & YM \\
\hline Sexo & 0,184 & 0,295 & 0,391 & 0,789 & 0,467 & 0,319 & 0,331 & 0,844 \\
Escolaridade & 0,250 & 0,726 & 0,518 & 0,645 & 0,769 & 0,882 & 0,636 & 0,627 \\
Estrato & 0,141 & 0,305 & 0,351 & 0,741 & 0,161 & 0,332 & 0,862 & 0,586 \\
Econômico & & & & & & & & \\
\hline
\end{tabular}

Valores de "p" obtidos pelo teste do Qui-quadrado.

\section{Discussão}

O principal objetivo deste estudo foi comparar a qualidade de vida, auto estima e auto imagem dos descendentes japoneses das colônias de Santa Catarina (Brasil) com os residentes da província de Yamanashi (Japão). Como são escassos os estudos que investigam a qualidade de vida e a auto estima e auto imagem de descendentes, buscamos como ponto de partida para

Pensar a Prática, Goiânia, v. 14, n. 1, p. 1-12, jan./abr. 2011 
discussão, estudos que abordam as mesmas variáveis mesmo sendo em populações diferentes, e mais especificamente, estudos que tenham utilizado os mesmo instrumentos na coleta de dados.

A auto estima e auto imagem foi considerada baixa para praticamente todos os participantes do estudo, sendo que a situação mostrou-se mais delicada para os descendentes residentes em Yamanashi. Os escores da qualidade de vida dos descendentes de Santa Catarina são levemente mais altos que os descendentes de Yamanashi, mas não apresentam diferenças estatísticas.

No estudo de Bittencourt e Hoenhne (2009) que utilizaram o mesmo instrumento apresenta resultados semelhantes no que diz respeito à qualidade de vida conceituada como boa. No entanto existem estudos também com o mesmo instrumento que contrariam a afirmação, apresentando resultados de qualidade de vida baixa (ALVES e COL., 2009; ARAGÃO e COL., 2009). De acordo com a hipótese inicial do estudo, de que talvez a qualidade de vida fosse diferente entre os grupos de descendentes japoneses, encontrou-se que o domínio psicológico apresentou diferença entre os descendentes de Santa Catarina e Yamanashi. Acredita-se que estes escores inferiores no domínio psicológico dos descendentes residentes em Yamanashi possam estar relacionados ao fato de estarem residindo longe de casa, de seus familiares e amigos, além do convívio social com pessoas que falam outro idioma e possuem outra cultura como no estudo de Wang e col., (2000), que comparou a qualidade de vida de adolescentes japoneses e chineses. Similarmente, Tamayo e col (2001) investigando também a população adulta obtiveram diferença estatística no domínio psicológico. A possibilidade de contar com rede de apoio, senso de coesão de grupo, aceitação da própria aparência e atribuição de valor à vida são fatores que protegem a saúde mental, evitando quadros de insônia ou irritabilidade, ansiedade e depressão (ADEODATO e COL., 2005).

Acrescenta-se que no período de coleta de dados as grandes forças econômicas mundiais, entre as quais destaca-se o Japão, estavam enfrentando uma grave crise. Em situações como estas os setores produtivos são amplamente afetados, o que pode ter desencadeado insegurança e frustrações dos descendentes em Yamanashi. O domínio físico (trabalho, dor, desconforto, energia, fadiga, sono e repouso) foi o de menor escore nos dois locais seguido pelo domínio psicológico (sentimentos positivos, pensar, aprender, memória, concentração, auto estima, imagem corporal, sentimentos negativos, depressão e ansiedade). Talvez, isto possa ter ocorrido pelas condições de trabalho encontradas no Japão, que de acordo com Penteado e Pereira (2007) em seu estudo com adultos utilizando o mesmo instrumento, observaram que um ambiente de trabalho com condições precárias causa impacto negativo que consumirá energias para a capacidade do trabalho e atividades rotineiras. A qualidade de vida nesse estudo foi considerada boa de uma forma geral, principalmente no domínio social, que segundo Souza e col. (2003) depende em parte de outras questões como relações interpessoais, familiares e sexuais.

No estudo de Bosi e col. (2006) a auto estima e auto imagem também obtiveram conceituação baixa, corroborando com os resultados apresentados neste estudo, diferentemente de Vargas e col. (2005), no qual os escores de adultos foram mais altos. O aumento ou diminuição da auto estima traz uma elevada carga emocional que podem estar ligadas ao sucesso ou não na vida (BAUMEISTER e COL., 2003; NOVATO e COL., 2008), podendo também estar relacionada com a felicidade que influenciará na qualidade de vida.

Num estudo utilizando o mesmo instrumento com adultos, a prática de atividade física regular contribui na melhora da saúde física e mental, consequentemente na elevação da auto

Pensar a Prática, Goiânia, v. 14, n. 1, p. 1-12, jan./abr. 2011 
estima e auto imagem, influenciando na qualidade de vida (MAZO e COL., 2006). Para atender mais adequadamente estas situações deve-se abordar a atividade física direcionada mais à saúde e funcionalidade do que para o condicionamento físico, como descrito na literatura (MCAULEY e COL., 2005). Nesse estudo a prática de atividade física foi bastante esporádica não atingindo a recomendação para promoção da saúde, a qual consiste em uma prática de intensidade pelo menos moderada, cinco vezes por semana no mínimo trinta minutos por dia (150 min/semana) ou, em intensidade vigorosa três dias por semana e quinze minutos por dia (75 min/semana) (HASKELL e COL., 2007). O nível de atividade física pode variar de acordo com o gênero, idade, nível socioeconômico e região, podendo influenciar positivamente a qualidade de vida (TAMAYO e COL., 2001; PENTEADO e PEREIRA, 2007).

Quanto à influência da cultura japonesa $86,4 \%$ dos participantes declararam que a cultura foi positiva em sua vida, como ressalta Takeuchi (2008) em seu estudo com população de mesma origem étnica. Os descendentes foram educados com uma mistura da influência cultural dos brasileiros, mas nunca esquecendo suas origens. Entretanto Wang e col (2000) salientam que não há uma relação direta da qualidade de vida com a cultura, há sim uma certa influência por parte do momento em que o país de origem está passando, pelas situações políticas e econômicas.

\section{Conclusão}

Percebe-se neste estudo que o domínio psicológico foi o único indicador da qualidade de vida que obteve diferença entre os descendentes de Santa Catarina e Yamanashi, talvez pela distância da família, do país de origem e dos amigos. A auto estima e auto imagem foram consideradas baixas, por praticamente todos os descendentes japoneses. Sugerindo-se que uma aderência à prática de atividade física possa, por meio de contato com novas pessoas, aumentar os escores da qualidade de vida de boa para excelente e, principalmente aumentar a auto estima e auto imagem destes indivíduos.

\section{REFERÊNCIAS}

ABEP - Associação Brasileira de Empresas de Pesquisa. Critério Padrão de Classificação Econômica Brasil/2008. Disponível em: http://www.abep.org/?usaritem=arquivos\&iditem=23. Acesso em: 20/12/2008 às 20:30:32.

ADEODATO, V.G.; CARVALHO, R.R.; SIQUEIRA, V.R.; SOUZA, F.G.M. Qualidade de Vida e Depressão em Mulheres Vítimas de seus Parceiros. Revista de Saúde Pública, São Paulo, v. 39, n,1, p. 108-113, jan. 2005.

ALVES, A.L.A.; SALIM, F.M.; MARTINEZ, E.Z.; PASSOS, A.D.C.; DE CARLO, M.M.R.P.; SCARPELINI, S. Qualidade de Vida de Vítimas de Trauma Seis Meses Após a Alta Hospitalar. Revista de Saúde Pública, São Paulo, v.43, n.1, p. 156-160, fev. 2009.

ALVES, J.G.B; MONTENEGRO, F.M.U; OLIVEIRA, F.A; ALVEZ, R.V. Prática de Esportes

Pensar a Prática, Goiânia, v. 14, n. 1, p. 1-12, jan./abr. 2011 
Durante a Adolescência e Atividade Física na Vida Adulta. Revista Brasileira de Medicina do Esporte, v.11, n.5, p. 291-294, 2005.

ARAGÃO, A.T.M.; MILAGRES, E.; FIGLIE, N.B. Qualidade de Vida e Desesperança em Familiares de Dependentes Químicos. Psico-USF, Itatiba, v.14, n.1, p.117-123, jan-abr, 2009.

BARBETTA, P.A. Estatística Aplicada às Ciências Sociais. 6.ed.rev. Florianópolis: Ed. Da UFSC, 2006. 315p.

BAUMEISTER, R.F.; CAMPBELL, J.D.; KRUEGER, J.I.; VOHS, K.D. Does High Self-Esteem Cause Better Performance, Interpersonal Success, Happiness, Or Healthier Lifestyles?; Psychological Science in the Public Interest, v. 4, n.1, p. 1-44, 2003.

BITTENCOURT, Z.Z.L.C.; HOENHNE, E.L. Qualidade de Vida de Familiares de Pessoas Surdas Atendidas em um Centro de Reabilitação. Ciência e Saúde Coletiva, Rio de Janeiro, v.14, n.4, p. 1235-1239, jul-agos 2009.

BOSI, M.L.M; LUIZ, R.R; MORGADO, C.M.C; COSTA, M.L.S; CARVALHO, R.J. Auto Percepção da Imagem Corporal entre Estudantes de Nutrição: Um Estudo no Município do Rio de Janeiro. Revista Brasileira de Psiquiatria, v.55, n.2, p. 108-133, 2006.

Censo Demográfico de Santa Catarina. Instituto Brasileiro de Geografia - IBGE, 1940, 1955, 1960, 1970, 1980 e 1991.

FLECK, M.P.A; LOUZADA, S; XAVIER, M; CHACHAMOVICH, E; VIEIRA, G; SANTOS, L; et al. Aplicação da versão em português do instrumento de avaliação de qualidade de vida da OMS. (Whoqol - 100). Revista de Saúde Pública, v. 33, n.2, p. 198-205, 1999.

FLECK, M.A; LIMA, A.F.B.S; POLANCZYK, C.A; TRENTINI, C.M; BANDEIRA, D; PATRICK, D; et al. A avaliação de qualidade de vida: guia para profissionais da saúde. Porto Alegre: Artmed, 2008.

HASKELL, W.L; LEE, I.M; PATE, R.R; POWELL, K.E; BLAIR, S.N; FRANKLIN, B.A; MACERA, C;A, HEATH, G.W; THOMPSON, A.B. Physical activity and public health: updated recommendation for adults from the American College of Sports Medicine and the American Heart Association. Medicine and Science of Sports Exercise, Special reports, p.1423-1434, 2007.

MARTINELlO, A.S; KLUG, J. Política Agrária e Imigratória nas Colônias Japonesas de Santa Catarina (1961 - 1978). Monografia de graduação. Florianópolis: UFSC, CFH; 2007.

MAZO, G.Z.; CARDOSO, F.L.; AGUIAR, D.L. Programa de Hidroginástica para Idosos: motivação, auto estima e auto imagem. Revista Brasileira de Ciência e Desenvolvimento

Pensar a Prática, Goiânia, v. 14, n. 1, p. 1-12, jan./abr. 2011 
10

10.5216/rpp.v14i1.12368

Humano, v. 8, n. 2, p. 143-174, 2006.

McAULEY, E.; ELAVSKY, S.; MOTL, R.W.; KONOPACK, J.F.; HU, L.; MARQUEZ, D.X. Physical Activity, Self Efficacy and Self Esteem: Longitudinal relationship in older adults. Journal of Gerontology: Psychological Science, v.60B, n.5, p. 268-275, 2005.

NOVATO, T.S.; GROSSI, S.A.A.; KIMURA, M. Qualidade de Vida e Auto Estima de Adolescentes com Diabetes Mellitus. Acta Paulista de Enfermagem. v.21, n.4, p.562-567, 2008

PENTEADO, R.Z.; PEREIRA, I.M.T.B. Qualidade de Vida e Saúde Vocal de Professores. Revista de Saúde Pública. v.41, n.2, p. 236-243, 2007.

PINHEIRO, S.E.M.; SUAREZ, M.T.S.M. Ser ou Não Ser Japonês? A construção da identidade dos brasileiros descendentes de japoneses no contexto das migrações internacionais do Japão Contemporâneo. Tese de Doutorado, Campinas, SP, 2009.

SOUSA, L.; GALANTE, H.; FIGUEIREDO, D. Qualidade de Vida e Bem Estar dos Idosos: Um estudo exploratório na população portuguesa. Revista de Saúde Pública, v.37, n.3, p. 364-71, 2003.

STEGLICH, L.A. Terceira Idade, Aposentadoria, Auto Imagem e Auto estima. Dissertação de Mestrado. UFRGS, Porto Alegre RS, 1978.

TAKEUCHI, M.Y. A Comunidade Nipônica e a Legitimação de Estigmas: O Japonês Caricaturizado. Revista da USP, n.79, p.173-82, 2008.

TAMAYO, A., CAMPOS, A.P.M.; MATOS, D.R.; MENDES, G.R.; SANTOS, J.B.; CARVALHO, N.T. A Influência da Atividade Física Regular Sobre o Autoconceito. Estudos de Psicologia, v.2, p.157-165., 2001

WANG, X.; MATSUDA. N.; MA, H.; SHINFUKU, N. Comparative Study of Quality of Life Between the Chinese and Japanese Adolescent Populations. Psychiatry and Clinical Neurosciences, v.54, p. 147-152, 2000.

VARGAS, T.V.P.; DANTAS, R.A.S.; GOIS, C.F.L. A Auto Estima de Indivíduos que Foram Submetidos à Cirurgia de Revascularização do Miocárdio. Revista da Escola de Enfermagem USP, v.39, n.1, p.20-27, 2005.

NAGEL, G.; SILVA, R.M.; SAKAE, T.M.; MIRANDA, E.P.; VARGAS, F.R.; MORETTI, G.R.S.; ALMEIDA, G.K.P.; ALMEIDA, L.P.; FORNASA JÚNIOR, L.C.; MORI, L.K. Qualidade de vida no município de Tubarão - SC, segundo a escala de qualidade de vida de Flanagan. Arquivos Catarinenses de Medicina, v. 38, n. 2, p.29-36, 2009.

ALEXANDRE, T.S.;CORDEIRO, R.C.; RAMOS, L. R. Factors associated to quality of life in Pensar a Prática, Goiânia, v. 14, n. 1, p. 1-12, jan./abr. 2011 
11

10.5216/rpp.v14i1.12368

active elderly. Revista de Saúde Pública, v.43, n.4, p. 613-621, 2009.

COQUEIRO, R.S.; PETROSKI, E.L.; PELEGRINI, A.; BARBOSA, A.R. Insatisfação com a imagem corporal: avaliação comparativa da associação com estado nutricional em universitários. Revista de Psiquiatria do Rio Grande do Sul, v.30, n.1, p. 31-38, 2008.

KAKESHITA, I.S.; ALMEIDA, S.S. Relação entre índice de massa corporal e a percepção da auto-imagem em universitários. Revista de Saúde Pública, v.40, n.3, p. 497-504, 2006.

THE WHOQUOL GROUP. The world health organization quality life assessment (WHOQUOL): Position paper from the world health organization. Social Science e Medicine, v.41, n.10, p.1403 -1409, 1995.

SANTOS, S.R.; SANTOS, I.B.C.; FERNANDES, M.G.M.; HENRIQUES, M.E.R.M. Elderly quality of life in the community: application of the Flanagan's Scale. Revista Latino-Americana de Enfermagem, v.10, n.6, p.757-764, 2002.

VELARDE, J.E.; AVILA, F.C. Methods for quality of life assessment. Salud Pública de México, v.44, n.4, p.349-361, 2002.

BOWLING, A.; GABRIEL, Z.; DAKES, J.; DOWDING, L.M.; EVANS, O.; FLEISSIG, A. ET AL. Let's ask them: a national survey of definitions of quality of life and its enhancement among people aged 65 and over. International Journal of Aging Human Development, v.56, n.4, p.269-306, 2003.

PEREIRA, N.B.R.; DUVICQ, C.G.F.; CARVALHO, A.M.P. Influencia del consumo de sustancias psicoactivas en el ámbito familiar sobre la autoestima de escolares. Revista LatinoAmericana de Enfermagem, v.13, num esp., p.798-805, 2005.

\section{QUALITY OF LIFE, SELF ESTEEM AND SELF IMAGE OF JAPANESE COLONIES FROM BRAZIL AND JAPAN}

Abstract: Objective: To analyse and compare the quality of life (QL), self-esteem, and self image of Japanese descendents from Santa Catarina (SC) and from Yamanashi. Methods: A

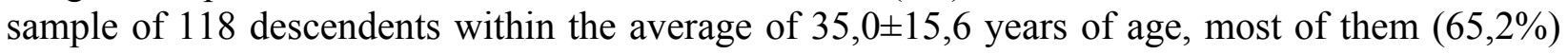
living in SC (Brazil). Results: The participants live by themselves; have paid work and incomplete undergraduate course. The QL was considered from good to very good (56-72\%), the descendents from SC had higher scores, with meaningful difference in the psychological domain $(p=0,050)$. The self-esteem, self image had lower concept. Besides that, they do not have the habit of regular physical activity (PA) practice. Conclusion: The self esteem and self image were considered low, and QL good, not differing between the groups in general.

Pensar a Prática, Goiânia, v. 14, n. 1, p. 1-12, jan./abr. 2011 
12

10.5216/rpp.v14i1.12368

Keywords: Japanese, Quality of Life, Corporal Image, Self Image

\section{CALIDAD DE VIDA, AUTOESTIMA Y AUTOIMAGEN DE JAPONESES DE BRASIL Y JAPÓN}

Resumen: Objetivo: Analizar y comparar la calidad de vida (CV), autoestima, y autoimagen de los de descendientes japoneses de Santa Catarina (SC) y de Yamanashi. Metodos: Una muestra de 118 descendientes dentro del promedio de los 35,0土15,6 años de edad, la mayoría (el 65.2\%) viviendo en SC (Brasil). Resultados: Los participantes viven solo y tienen enseñanza superior incompleta. La CV fue considerada de bueno a muy bueno (56-72\%), los descendientes de SC tuvieran score más alto, con diferencia significativa en el dominio psicológico $(p=0,050)$. El autoestima, y autoimagen tuvieran concepto más bajo. Además de eso, no tienen el hábito de la práctica regular de la actividad física (AF). Conclusión: La autoestima y autoimagen fueran consideradas bajas, y la CV buena, no diferenciando entre los grupos en general.

Palabras-clave: Japoneses, Calidad de Vida, Imagen Corporal, Autoimagen

Endereço para correspondência:

Zenite Machado

d2zm@udesc.br

Universidade do Estado de Santa Catarina

Centro de Educação Física e Desportos

Paschoal Simone, 358

Coqueiros

88080-350 - Florianopolis, SC - Brasil

Pensar a Prática, Goiânia, v. 14, n. 1, p. 1-12, jan./abr. 2011 